\title{
MEDITATION EEG INTERPRETATION BASED ON NOVEL FUZZY-MERGING STRATEGIES AND WAVELET FEATURES
}

\author{
KAng-Ming Chang ${ }^{1,2}$, PeI-Chen Lo ${ }^{1}$ \\ ${ }^{1}$ Department of Electrical and Control Engineering, \\ National Chiao Tung University, Taiwan \\ ${ }^{2}$ Department of Computer and Communication Engineering, Asia University, Taiwan
}

\begin{abstract}
As the advantages of meditation have been outlined literally, scientific exploration of the meditation phenomena becomes significant. Meditation EEG may provide an access to the mental states beyond normal consciousness. It is the first attempt to score the meditation course by EEG. Wavelet analysis and fuzzy c-means (FCM) are applied in the automatic interpretation algorithm. However, FCM applied straightforward to quantitative feature vectors often results in an over-trifling interpretation. As a consequence, this paper presents novel cluster-managing strategies for achieving an interpretation closer to the result of naked-eye examination. The running gray-scale chart, derived by extracting, clustering, and coding the EEG features, reveals five different meditation scenarios differing from those of the controlled subjects.
\end{abstract}

Biomed Eng Appl Basis Comm, 2005(August); 17: 167-175.

Keywords: Meditation EEG (electroencephalogram) scoring, Wavelet analysis, Fuzzy c-means clustering $(\mathrm{FCM})$, meditation scenario.

\section{INTRODUCTION}

Zen-Buddhist meditation originated more than 2,500 years ago, and had been proved to benefit the health while on the way toward the ultimate "Buddhahood" state. Meditation process reflects a brain state completely differing from the normal consciousness or the sleep states. Different meditating techniques have been studied for several decades [1][12]. They are mostly the TM (transcendental meditation), Yoga, and Japanese Zen meditation, with

Received: Jan. 7, 2005; Accepted: July 15, 2005

Correspondence: Pei-Chen Lo, Professor

Department of Electrical and Control Engineering,

National Chiao Tung University, 1001 Ta-Hsueh Road,

Hsinchu 30010, Taiwan

E-mail: pclo@faculty.nctu.edu.tw the focus mainly on the physiological and psychological effects of meditation. This paper presents a systematic approach for meditation EEG interpretation and firstly reports the results of investigating meditation scenarios of the orthodox Zen-Buddhist practitioners.

A number of papers have reported the EEG findings for experimental subjects under meditation [3]-[5] [8]-[12]. EEG has been recognized to be an important clinical tool for diagnosing and monitoring the nervous system [13]-[15]. In the meditation EEG study, West [5] summarized the EEG findings and made three major comments including: slower alpha with larger power at the meditating beginning, occurrence of the rhythmic theta trains for experienced meditators at the mid session, and very rarely, bursts of high-frequency beta (above $20 \mathrm{~Hz}$ ) observed for 
meditators capable of achieving deep meditation, the so-called samadhi or transcendence. Thus, it was suggested that the beta-dominated pattern characterized the EEG under deep 4 meditation stage.

In spite of the extensive study on meditation EEG since 1960 , no report was found regarding the meditation scenario based on the EEG features. This paper proposes a strategy of establishing an overview of the meditation EEG record. The strategy involves a robust approach in consideration of the inter-subject variation. We extract the subject-oriented EEG patterns in meditation, without a pre-specified, prototypical base, to characterize the meditation process. The approach mainly applies the FCM clustering to the feature vectors derived from the wavelet coefficients. Thus, the feature basis is oriented towards the particular EEG patterns of each individual meditator.

The following section introduces the experiment, the method of feature quantification, and the strategies for meditation-EEG interpretation. Section III discusses the interpreting results with relation to possible meditation scenarios depicted by the meditators. Section IV makes a remark on this preliminary study.

\section{METHOD}

\section{A. Experimental protocol}

The meditation EEG signals were recorded using 8-channel SynAmps amplifiers (manufactured by NeuroScan, Inc.) connected to the Pentium MMX-166 $(\mathrm{MHz}) \mathrm{PC}$. We applied the 8-channel unipolar recording montage of which the common reference was the linked MS1-MS2 (mastoid electrodes). The 8channel EEG electrodes were placed at F3, F4, C3, C4, $\mathrm{P} 3, \mathrm{P} 4, \mathrm{O} 1$, and $\mathrm{O} 2$. The sampling rate was $400 \mathrm{~Hz}$. Each recording lasted for 45 minutes, including the first 5-minute background EEG (the subject sat in normal relaxed position with eyes closed) and the rest 40-minute meditation EEG. During the meditation session, the subject sat, with eyes closed, in the fulllotus or half-lotus position. Each hand formed a special mudra (called the Grand Harmony Mudra), laid on the lap of the same side. The subject focused on the Zen Chakra and the Dharma Eye Chakra (also known as the "Third Eye Chakra" ) in the beginning of meditation till transcending the physical and mental realm. The Zen Chakra locates inside the third ventricle, while the Dharma Eye Chakra locates at the hypophysis.

\section{B. Feature vector derived from wavelet coefficients}

For the past two decades, Wavelet analysis has been extensively studied and proved to be a useful tool in biomedical signal processing [16]-[20]. Appropriate selection of scales and wavelet bases enables it to characterize the EEG rhythmic patterns [20]-[22]. According to our study, wavelet prototype of appropriate duration has little effect on the quantitative feature vector. We employ db5 wavelet prototype in this study. In consideration of computational efficiency, the discrete Wavelet 6 transform (DWT) is often applied. As illustrated in table 1, the DWT scales D4 $\sim$ D7 are approximately matched to those welldefined EEG rhythmic bands [20]-[22], assuming a sampling rate of $400 \mathrm{~Hz}$. The feature vector is thereafter constructed from these DWT coefficients. The procedure is depicted below.

Firstly, the 2-second running window, moving at a step size of 1 second, is employed. And the entire meditation EEG record is divided into $L$ segments. Consider a discrete-time signal $x[n], 0 \leq n \leq N-1$ $(N=800)$, representing the $l$ th running EEG epoch. The $a_{j}[n]$ and $d_{j}[n]$ indicate, respectively, the coarse and detailed sequences after $j$ 's decompositions. They can be obtained by

$$
\begin{aligned}
& a_{j}[n]=\sum_{k=-\infty}^{\infty} a_{j-1}[k] \cdot h[2 n-k], \text { and } \\
& d_{j}[n]=\sum_{k=-\infty}^{\infty} a_{j-1}[k] \cdot g[2 n-k],
\end{aligned}
$$

where $a_{0}[n]=x[n], h[n]$ is the scaling filter, and $g[n]$ is the wavelet filter. The lth running feature vector, $v_{k}[l]$, is extracted from the selected detailedscale coefficients by computing their powers as

$$
v_{k}[l]=\frac{1}{n_{k}} \sum_{i=1}^{n_{k}} d_{k}^{2}[i] \quad k=4,5,6,7,
$$

where $n_{k}$ is the length of $d_{k}$. The feature vector of the lth EEG epoch accordingly is

$$
v[l]=\left\{v_{4}[l], v_{5}[l], v_{6}[l], v_{7}[l]\right\} .
$$

Finally, $\underline{V}=\{[l]] 0 \leq l \leq L-1\}^{T}=\left\{\left[[0] \ldots, v[L-1]^{T}\right.\right.$ is an L $\times 4$ feature matrix of which each row indicates the running feature vector.

\section{FCM-merging strategies}

Automatic interpretation algorithm often involves three strategies: derivation of feature basis, feature clustering, and scoring (interpretation) based on the feature clusters. Feature extraction aims at transforming the input data into a form (feature vector) appropriate for the clustering algorithm to identify the clusters. The feature vector in this study is derived from wavelet coefficients. Each feature vector, after 
processed by the FCM, belongs to a cluster to some degree that is specified by a membership matrix. Jim Bezdek originally introduced this technique [23]. According to our experience in EEG feature classification, conventional FCM algorithm, without the background knowledge of EEG characteristics, cannot effectively classify and interpret the EEG record in comparison with the naked-eye examination. We thus developed a novel approach, with three cluster-merging strategies, for the meditation EEG analysis. The main attribute is its knowledge-based processing of EEG record that is encoded into an easily comprehensible chart of meditation scenario. Fig. 1(a) illustrates the overall strategy developed according to our experiences on meditation EEG characteristics. The FCM-merging strategies involving three clustermerging subroutines (Fig. 1(b), 1(c), and 1(d)) are designed particularly to solve the problem 8 of blind clustering by simple FCM algorithm. Cluster-merge A (Fig. 1(b)) mainly determines the number of clusters $(K)$ by the criterion of cluster-center distance $\left(\mathrm{D}_{i j}\right)$. Note that the number of clusters is often initialized to be larger than that required.

Further processing of clusters thus does not involve splitting. Cluster-merge B (Fig. 1(c)) eliminates those clusters characterizing transient activities. Finally, cluster-merge C (Fig. 1(d)) assigns different clusters, actually representing the same EEG rhythm with amplitude fluctuation, to be the same one. We first describe the FCM function in subroutines cluster-merge $\mathrm{A}$ and $\mathrm{B}$. The FCM function analyzes the EEG data and produces three outputs: (1) the membership matrix $\underline{\boldsymbol{U}},(2)$ the cluster centers $\{\mathrm{c} i, i=1$, $\cdots, \mathrm{K}\}$, and (3) the EEG coding vector (row matrix) $\underline{\boldsymbol{S}}$. Four steps in the FCM function are depicted below.

Step 1: Initialize the membership matrix $\underline{\boldsymbol{U}}$ with random values between 0 and 1 . The size of $\underline{U}$ is $\mathrm{K} X$ $\mathrm{L}$, where $K$ is number of clusters, and $L$ is the number of input feature vectors. The element of membership matrix $\underline{\boldsymbol{U}}, \mathrm{u}_{i j}$, is the probability that the $j$ th feature vector belongs to the ith cluster $(1 \leq i \leq \mathrm{K}$ and $0 \leq j \leq \mathrm{L}-1)$. Note that, for a given feature vector, summation of degrees of belongness equals unity. Elements of $U$ must satisfy the constraint below

$$
\sum_{i=1}^{K} u_{i j}=1, \quad 0 \leq j \leq L-1 .
$$

Step 2: Calculate fuzzy cluster centers for all $K$ clusters according to

$$
c_{i}=\frac{\sum_{j=0}^{L-1} v[j] \cdot u_{i j}}{\sum_{j=0}^{L-1} u_{i j}}, \quad 1 \leq i \leq K,
$$

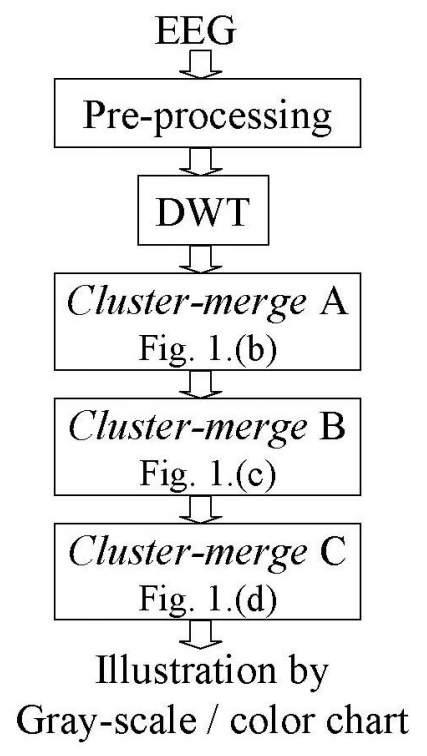

Fig.1(a)

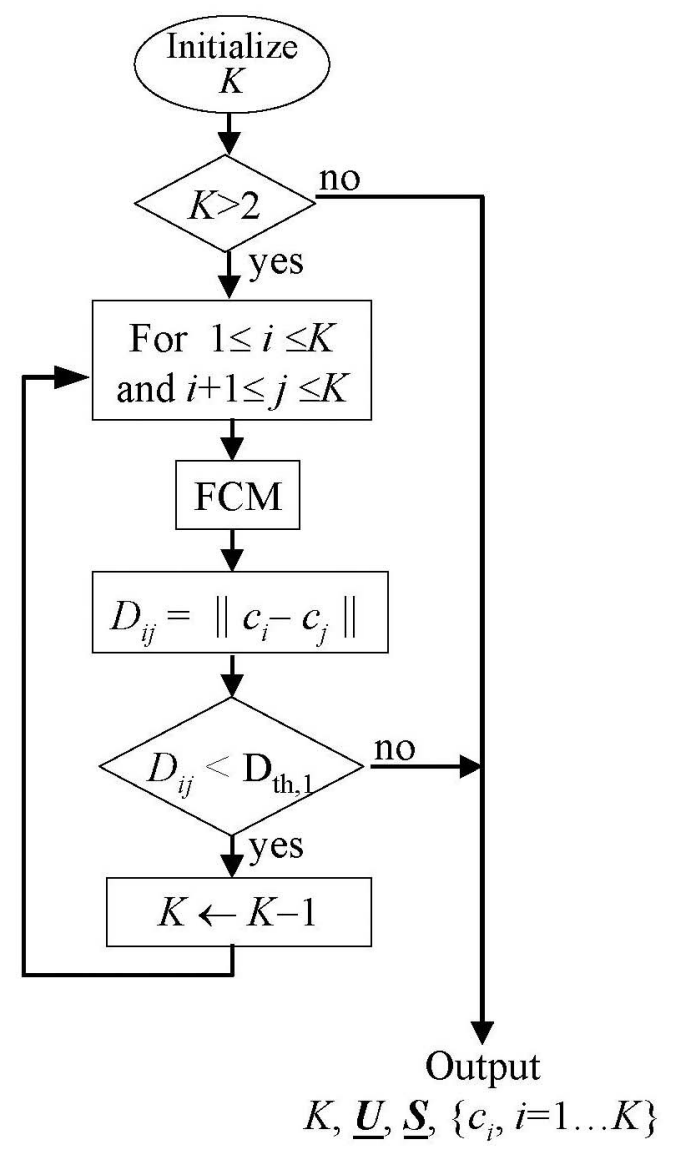

Fig.1(b) 


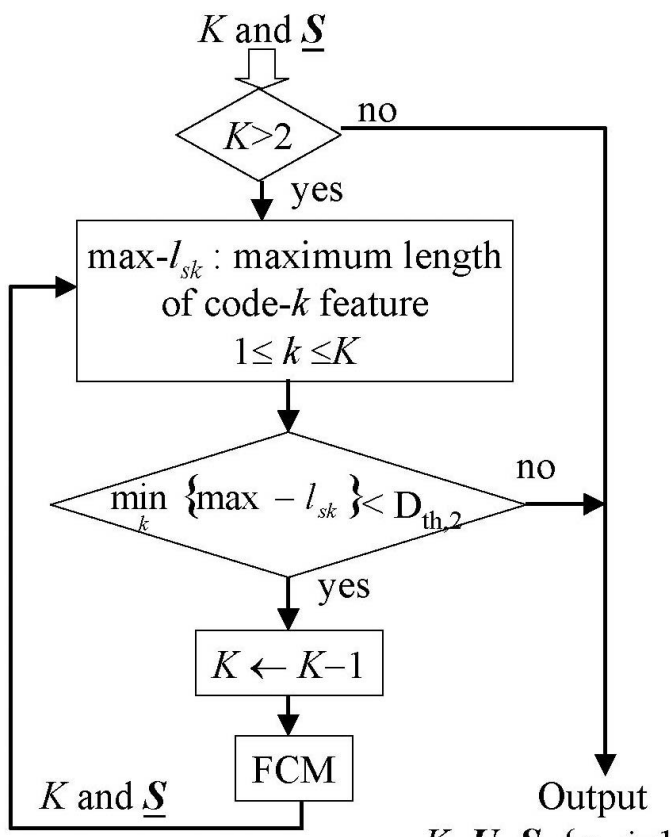

$K, \underline{\boldsymbol{U}}, \underline{\boldsymbol{S}},\left\{c_{i}, i=1 \ldots K\right\}$

Fig.1(c)

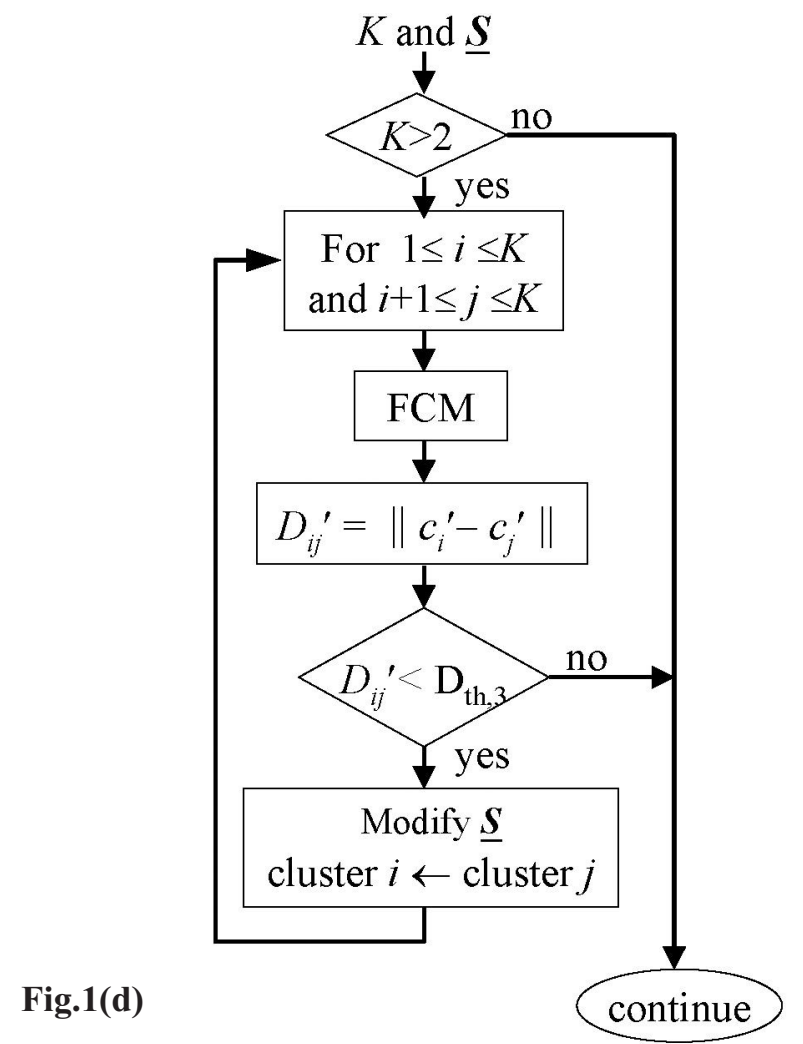

Fig.1 The block diagram of FCM-merging strategies (1(a) involve three cluster-merging subroutines (1(b), 1(c), and 1(d)). where $v[j]$ is the feature vector $(1 \times 4$ row vector $)$ derived from wavelet coefficients defined in (4).

Step 3: Compute the cost function defined below

$$
J\left(\underline{U}, c_{1}, c_{2, \ldots}, c_{K}\right)=\sum_{i=1}^{K} J_{i}=\sum_{i=1}^{K} \sum_{j=0}^{L-1} u_{i j}^{m} D_{i j}^{2},
$$

where $D_{i j}=\| c_{i}-v[j]$ is the Euclidean distance between the $i$ th cluster center and the $j$ th feature vector. The weighting exponent, $m$, in (7) is selected to be $m=2$. The criterion requires $J$ to be as small as possible. The iteration terminates if improvement of $\boldsymbol{J}$ over previous iteration is below a pre-specified threshold. Otherwise, the algorithm proceeds with next step to update the membership matrix $\underline{\boldsymbol{U}}$.

Step 4: Compute a new membership matrix $\underline{\boldsymbol{U}}$ whose element $u_{i j}$ is adjusted by

$$
u_{i j}=\frac{1}{\sum_{k=1}^{K}\left(\frac{D_{i j}}{D_{k j}}\right)^{2 / m-1}}, \quad 1 \leq i \leq K, \quad 0 \leq j \leq L-1 .
$$

According to the above equation, $u_{i j}$ is inversely proportional to the squared distance from the feature $v[j]$ to the cluster center $c_{i}$. The iteration process from Step 2 to Step 4 continues.

The FCM function described above blindly classifies the EEG patterns based on 10 quantitative features. Consequently, the result of interpretation often appears to be away from satisfaction. We accordingly developed sophisticated cluster-managing strategies, the FCM-merging strategies, based on background knowledge of meditation EEG characteristics, for achieving an interpretation closer to the result of naked-eye examination. We first generate a $1 \times L$ long-code row vector matrix, $\underline{\boldsymbol{S}}=\left\{\mathrm{s}_{i}, 0 \leq j \leq \mathrm{L}-1\right\}$, representing the encoded stream of $L$ feature vectors. Each element ( $\mathrm{s}_{i} \in$ integer) indicates, among $K$ clusters, the particular cluster to which the $j$ th feature vector belongs, $1 \leq \mathrm{s}_{i} \leq \mathrm{K}$. It is determined by finding the row index $i$ (denoted by $\mathrm{s}_{j}$ ) such that $u_{s, j}=\max \left\{u_{i j}\right\}, 1 \leq i \leq \mathrm{K}$, considering the $j$ th feature vector.

In the cluster-merge A subroutine (Fig. 1(b)), number of clusters $(K)$ is justified by having the inter-distance between cluster centers $\left(D_{i j}\right)$ no less than a pre-specified threshold $D_{\text {th }, 1}$. Four outputs available are: number of clusters $(K)$, membership matrix $(\underline{\boldsymbol{U}})$, EEG coding vector $(\underline{\boldsymbol{S}})$, and the cluster centers $\left\{\mathrm{c}_{i}\right.$, $1 \leq i \leq K\}$, of which only $K$ and $\underline{\boldsymbol{S}}$ feed into the clustermerge B subroutine (Fig. 1(c)). Based on the coding vector $\underline{\mathbf{S}}$, cluster-merge B first analyzes the maximum length of code- $k$ feature $(1 \leq k \leq K)$, max- $l_{\text {sk }}$. The length of code- $k$ feature $\left(l_{s k}\right)$ denotes the number of consecutive code $k$ 's. Consider an example of three clusters $(K=3)$ obtained by analyzing 18 feature vectors $(L=18)$. Assume the coding vector generated by 
cluster-merge A is:

$$
\underline{\boldsymbol{S}}=\{113333322111333322\},
$$

we then obtain three sets, each containing the segment lengths of code-k feature $(1 \leq k \leq 3)$ :

$$
\boldsymbol{l}_{\underline{\mathbf{s 1}}}=\{23\}, \boldsymbol{l}_{\underline{\mathbf{s 2}}}=\{22\}, \boldsymbol{l}_{\underline{\mathbf{s}}}=\{54\} \text {. }
$$

All the numbers in the $K$ sets are accordingly summed up to be $L$. From (10), the maximum length of code- $k$ feature is: $\max -l_{s 1}=3, \max -l_{s 2}=2$, and $\max -l_{s 3}=5$. If $\mathrm{D}_{\mathrm{th}, 2}=3$, cluster-merge $\mathrm{B}$ subroutine will decrease $K$ by 1 since $\min \left\{\max -l_{s k}\right\}=2<\mathrm{D}_{\mathrm{th}, 2}$. Otherwise, the last merging subrótine follows. After the operation of cluster-merge $\mathrm{B}$, the value of $K$ is more substantial and practical. The coding vector $\underline{S}$ is then derived using the final $K^{\prime}$ s, and both are fed into cluster-merge $\mathrm{C}$ subroutine. The goal of cluster-merge $\mathrm{B}$ is to eliminate the tedious work of interpreting those insignificant transient activities, which tend to complicate the result.

In EEG, considerable variation in amplitude often obscures identification of certain rhythmic pattern. For instance, FCM function tends to output multiple clusters for $\alpha$ rhythm classified according to the squared wavelet coefficients. This situation also occurs to $\Delta$ and $\theta$ rhythms. Cluster-merge $\mathrm{C}$ subroutine hence reexamines and corrects the fault by computing the subband power ratios as follows. Firstly, we modify the $\mathrm{L} \times 4$ feature matrix $\underline{\boldsymbol{V}}$ that is expressed by

$$
\underline{V^{\prime}}=\left\{v^{\prime}[0], v^{\prime}[1], \ldots, v^{\prime}[L-1]\right\}^{T},
$$

where $v^{\prime}[l]$ is the new $(1 \times 4)$ feature vector of the lth EEG epoch:

$$
v^{\prime}[l]=\left\{v_{4}^{\prime}[l], v_{5}^{\prime}[l], v_{6}^{\prime}[l], v_{7}^{\prime}[l]\right\}
$$
(4) by

Elements in $v^{\prime}[l]$ are derived from $v[l]$ in (3) and

$$
\begin{aligned}
& v_{k}^{\prime}[l]=\frac{v_{k}[l]}{v_{t}} \times 100 \%, 4 \leq k \leq 7, \text { where } \\
& v_{t}=\sum_{k=4}^{7} v_{k}[l] .
\end{aligned}
$$

Based on the modified feature matrix $\underline{\boldsymbol{V}^{\prime}}, \mathrm{FCM}$ function outputs a set of new cluster centers $\left\{c_{i}{ }^{\prime}\right.$, $1 \leq i \leq \mathrm{K}\}$. If cluster $j$ has a center $c_{j}{ }^{\prime}$ close enough to the center of cluster $i$ (i.e., $D_{i j}^{\prime}=\left\|c_{i}^{\prime}-c_{j}^{\prime}\right\|<D_{\text {th }, 3}$ ), the coding vector $\underline{\boldsymbol{S}}$ (output of cluster-merge B) will be modified by re-encoding cluster $\mathrm{j}$ as cluster $i$. In this way, different clusters actually containing feature vectors of the same EEG rhythm (e.g., $\alpha$ or $\theta$ ) are to be identified and interpreted as the same one via an adequate choice of $\mathrm{D}_{\text {th,3 }}$. It is noted that if the subband power ratios are employed in the very beginning (cluster-merge A), clustering performance is incorrigibly poor due to failure in separating different EEG rhythms. That is, cluster-merge $\mathrm{C}$ does not innovate upon the coding vector $\underline{\boldsymbol{S}}$. Instead, it re-indexes those clusters, all referred to the same EEG rhythm, by the same code.

In meditation EEG study, the range $2 \leq K \leq 5$ is a moderate selection according to our experience in meditation EEG interpretation. However, there always exists inter-subject variation in biomedical signals. Our algorithm thus begins with a large value of $K$ (normally, $K=9$ ) and, through the cluster-merging subroutines succeeded, further refines the interpreting results.

The FCM-merging strategies, systematically and effectively encoding the quantitative EEG features, were also proven to be robust to implementing parameters. Moreover, a wide range of mother wavelet prototypes can be used without changing the interpreting result should the wavelet duration be long enough. For instance, mother wavelets like the db3, $\mathrm{db} 5, \mathrm{db} 10, \mathrm{sym} 3$, and sym5 generated by MATLAB result in the same interpretation although the feature vectors derived midway are slightly different. We thus employed the mother wavelet $\mathrm{db} 5$ in consideration of computational efficiency.

\section{RESULTS AND DISCUSSION}

\section{A. Wavelet discrimination of EEG rhythm}

This sub-section is intended to justify the practicability of the proposed EEG feature vectors based on wavelet decomposition. We analyze a few simulated signals, each composed of four typical EEG rhythms. These EEG segments exhibit distinctive spectral peaks, and can be easily discriminated in the plot of wavelet coefficient powers. Fig. 2 illustrates the distribution of subband wavelet coefficient powers of four typical EEG rhythms. Four groups correspond to four wavelet coefficient powers, $v_{7}[\cdot], v_{6}[\cdot], v_{5}[\cdot]$, and $v_{4}[\cdot]$, with the length of bar indicating the range of quantifying feature $v_{k}[\cdot]$. In the $v_{k}[\cdot]$ group, the alpha, theta, delta, and flat patterns are symbolized respectively by dot, circle, asterisk, and square.

The feature vector formulated by wavelet coefficient powers thus provides a practical medium for distinguishing various EEG rhythmic patterns. As shown in Fig. 3, the $3 \mathrm{D}$ feature-vector space $\left\{v_{5}\left[{ }^{\cdot}\right]\right.$, $\left.v_{6}[\cdot], v_{7}[\cdot]\right\}$ demonstrates that feature vectors of the same EEG rhythmic pattern tend to gather together in the vector space. Although feature vectors of delta rhythm scatter over a larger region, further clustering-merging algorithm (FCM-merging strategies) may enhance the result of interpretation. 


\section{B. Five EEG patterns in meditation}

As normally characterized by its frequency, the EEG patterns are conveniently classified into four frequency ranges: the delta $(\Delta, f<4 \mathrm{~Hz})$, theta $(\theta$, $4 \mathrm{~Hz} \leq f<8 \mathrm{~Hz})$, alpha $(\alpha, 8 \mathrm{~Hz} \leq f \leq 13 \mathrm{~Hz})$, and beta $(\beta$, $f>13 \mathrm{~Hz}$ ). The meditation EEG signals, although composed of these standard rhythmic patterns, are found to orchestrate symphonies of certain tempos. After systematic study by applying the FCM-merging strategies to a number of meditation EEG data sets, results of clustering indicate that rhythmic patterns reflecting various meditation states normally involve five patterns: $\Delta, \Delta+\theta, \theta+$ slow $\alpha$ (denoted by $\alpha$ ), high-amplitude $\alpha$ (denoted by $\alpha^{+}$), and amplitudesuppressed wave. Fig. 4 displays the sample patterns. Among them, the EEG pattern of particular interest is the bottom one featured by "silent and almost flat" pattern. We denote this EEG pattern with the symbol “ $\Phi "$ in this paper [12].

Table 2 lists the feature vectors for the five EEG patterns in meditation. Those dominating coefficients of each EEG patterns are underlined. In $\Delta$ patterns, $v_{7}$ significantly dominates over the others, indicating that pure $\Delta$ can be successfully classified as one cluster by the FCM-merging strategies. Similarly, $\alpha^{+}$patterns has largest magnitude on the $v_{5}$ feature axis, which particularly characterizes the $\alpha$ band $(6.2 \sim 12.5 \mathrm{~Hz})$. The $\Delta+\theta$ patterns is characterized by two dominating

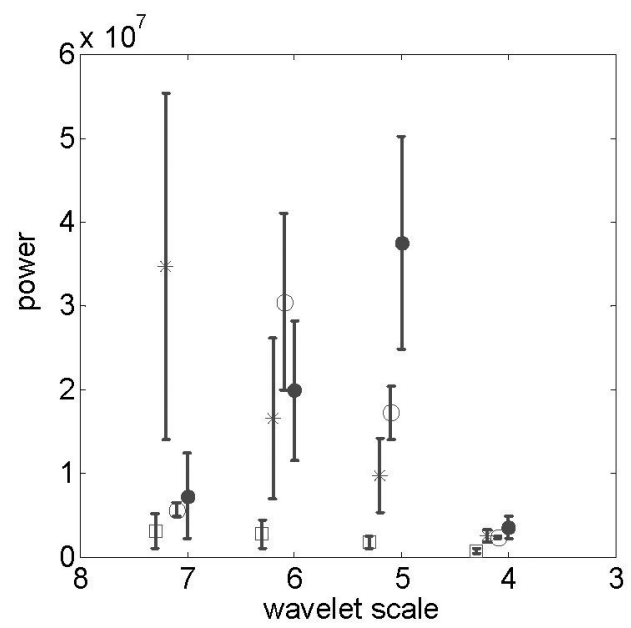

\begin{tabular}{|ll|}
\hline$*$ & alpha \\
* & theta \\
$*$ & delta \\
$\square$ & flat \\
\hline
\end{tabular}

Fig.2 Distribution of subband wavelet coefficient powers $\left(v_{7}, v_{6}, v_{5}\right.$, and $\left.v_{4}\right)$ of four typical EEG rhythms, $\Delta, \theta$, a, and $\beta$.
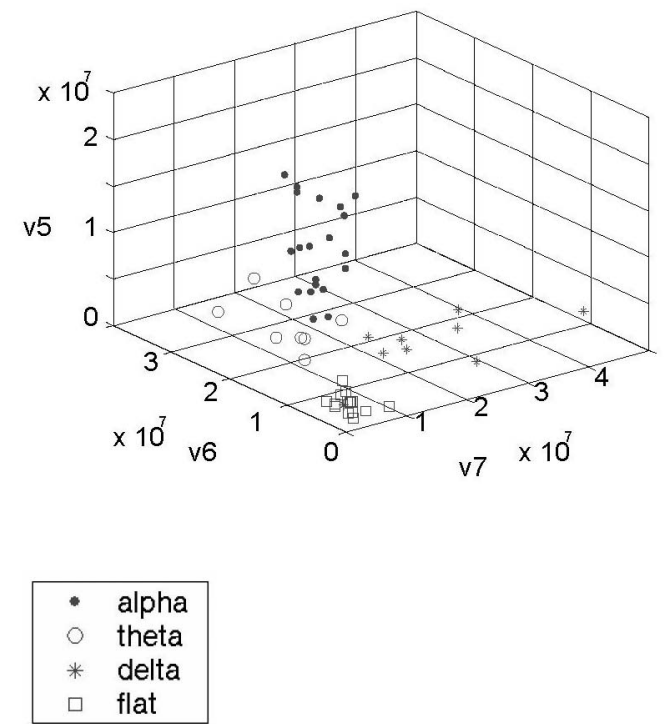

Fig.3 Three-dimensional illustration of the feature vector $\left\{v_{5}[\cdot], v_{6}[\cdot], v_{7}[\cdot]\right\}$ quantifying the $\alpha(\cdot), \theta$ $(\mathrm{O}), \Delta(*)$, and $\Phi(\square)$ activities.

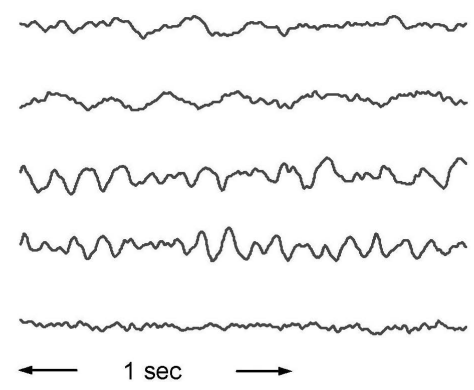

Fig.4 Five meditation-EEG patterns (from top): $\Delta$, $\Delta+\theta, \theta+\underline{\alpha}, \alpha^{+}$, and $\Phi$.

feature components, $v_{6}(3.1 \sim 6.2 \mathrm{~Hz})$ and $v_{7}(1.5 \sim 3.1$ $\mathrm{Hz}$ ), due to spectral intermixture of $\Delta$ and $\theta$. In other words, the wave shapes of both $\Delta$ and $\theta$ rhythms are not well-defined sinusoidal patterns. The $\theta$ and $\alpha$ activities often appears simultaneously, resulting in the $\theta+\alpha$ patterns. One unique cluster revealing particular meditation state transition is designated by the $\Phi$ patterns. Feature vectors in this cluster are characterized by (1) extremely low power (significant suppression of EEG amplitude), (2) corresponding temporal patterns with no particular EEG rhythm, and (3) no dominating peak in the spectral distribution. 
Table I : EEG rhythmic bands and the corresponding wavelet filter bands (sampling rate $f_{s}=400 \mathrm{~Hz}$ )

\begin{tabular}{|l|c|c|c|c|}
\hline EEG patterns & $\Delta$ & $\theta$ & $\alpha$ & $\beta$ \\
\hline Rhythmic bands $(\mathrm{Hz})$ & $1 \sim 4 \mathrm{~Hz}$ & $4 \sim 8 \mathrm{~Hz}$ & $8 \sim 13 \mathrm{~Hz}$ & $13 \sim 25 \mathrm{~Hz}$ \\
\hline Wavelet detail component $\left(D_{i}\right)$ & $\mathrm{D} 7$ & $\mathrm{D} 6$ & $\mathrm{D} 5$ & $\mathrm{D} 4$ \\
\hline Frequency bands of $(\mathrm{Hz})$ of $D_{i}$ & $1.5 \sim 3.1$ & $3.1 \sim 6.2$ & $6.2 \sim 12.5$ & $12.5 \sim 25.0$ \\
\hline
\end{tabular}

Table II: Subband wavelet power of five prototypes in meditation EEG.

\begin{tabular}{|c|c|c|c|c|}
\hline \multirow{2}{*}{ Feature vector of } & \multicolumn{4}{|c|}{ Subband power derived by $(3)\left(* 10^{\wedge} 7\right)$} \\
\cline { 2 - 5 } different prototypes & $v_{7}$ & $v_{6}$ & $v_{5}$ & $v_{4}$ \\
\hline$\Delta$ & $\underline{1.7044}$ & 0.4623 & 0.3586 & 0.1036 \\
\hline$\Delta+\theta$ & $\underline{0.9921}$ & $\underline{1.0543}$ & 0.1564 & 0.0669 \\
\hline$\theta^{+} \underline{\alpha}$ & 0.5658 & $\underline{3.0503}$ & $\underline{1.7233}$ & 0.2400 \\
\hline$\alpha^{+}$ & 0.1822 & 1.2312 & $\underline{2.2167}$ & 0.3866 \\
\hline$\Phi$ & 0.1115 & 0.1245 & 0.1164 & 0.0518 \\
\hline
\end{tabular}

\section{Five meditation EEG scenarios}

During the past five years, we have collected the EEG and other electrophysiological signals for more than fifty meditators. Their experiences on ZenBuddhism meditation range from a few months to sixteen years. Substantial meditation training leads them into the true Zen realm, that is, the spiritual world 16 beyond the Alaya consciousness (the $8^{\text {th }}$ consciousness). We thus are able to observe a variety of EEG changes during meditation session.

Different approaches for feature extraction may result in slight variation in interpretation of local, superficial EEG changes. Nevertheless, the quantitative illustration of whole meditation EEG record portrays a distinctive scenario for each particular meditator. To provide a long-term legible illustration, five meditation EEG patterns are displayed by different gray-scales. As illustrated in Fig. 5, the gray-scales from the darkest to the brightest colors indicate, respectively, the $\alpha^{+}, \Delta, \Delta+\theta, \theta+\underline{\alpha}$, and $\Phi$ patterns. Currently, five meditation scenarios have been observed based on evolution of meditation EEG illustrated by the running gray-scale chart. We present a summing-up report below.

Meditation scenario $A$ (Fig. 5(a))-- Persistent $\alpha$ activity dominates the entire meditation session. The
$\Phi$ activities of different duration appear intermittently. According to the narration of meditators in this group $(A)$, flights of thought continued throughout the meditation session. However, this kind of mental status would be abruptly transferred into an entirely peaceful, serene aura. This sensation happened, mostly accompanied with feeling of light, several times in one meditation course.

Meditation scenario $B$ (Fig. 5(b))-- Four EEG patterns, including $\alpha, \Delta, \theta$, and $\Phi$, appear rotationally. The meditator described the meditating sensation as follows: meditation status switched among sensations of (1) interference of mental activities, (2) alertness and tenseness, (3) subliminal status, and (4) feel of sacred, peaceful light.

Meditation scenario $C$ (Fig. 5(c))-- EEG signals of this group are characterized by background $\Phi$ with very few $\alpha$ activities. Scattered $\Delta$ 's are observed. The subject is a well-experienced meditator with more than ten-year Zen practicing. In the course of meditation, she felt the energy (or light) penetrating her head several times. At the moment of energy perception, her meditation became serene and egoless. She felt the liberation from her body and mind. Nonetheless, during the entire meditation course, thoughts or drowsiness might be in and out intermittently.

Meditation scenario $D$ (Fig. 5(d))-- Similar to the meditation scenario $C$, however, the background $\Phi$ is sprinkled by $\alpha$ instead of $\Delta$. The meditator experienced a bright (surrounded by the sacred light) and fully relaxed meditation in the test.

Meditation scenario E (Fig. 5(e))-- Their EEG's are unique. The $\Phi$ patterns dominates since the beginning of meditation, and no other activity is observed to be significant. Although the FCM-merging algorithm requires $K$ (number of clusters) to be larger than 2 , the "cluster $i \leftarrow$ cluster $j$ " operation in cluster-merge $\mathrm{C}$ further modifies the coding vector $\underline{\mathbf{S}}$ according to the subband power ratios, from which a uniform activity is identified. The interpreted result highly correlates with that of the naked-eye examination.

The running gray-scale chart derived from the controlled group tells quite another story. As shown in Fig. 5(f), the entire meditation scenario was dominated by the $\alpha$ rhythms, while $\Delta+\theta$ or $\theta$ appeared occasionally. Note that $\Delta$ and $\theta$ emerged because the subjects fell asleep. On the other hand, we observed, from the CCD video, the experimental subjects kept awake while $\Delta$ and $\theta$ emerged. This phenomenon aroused our attention. According to the subjects' narration, we hypothesized that $\Delta$ and $\theta$ activities are highly correlated with the subliminal consciousness. Another particular observation is the correlation between the occurrence of $\Phi$ patterns and the feeling of blessings by most experimental subjects. 


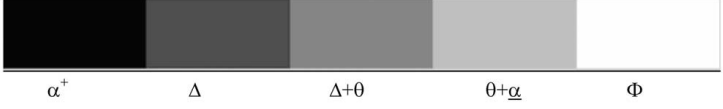

Fig. 5(a) Meditation scenario A

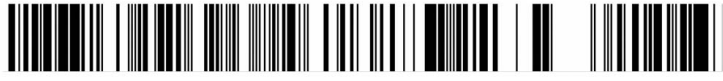

Fig. 5(b) Meditation scenario B

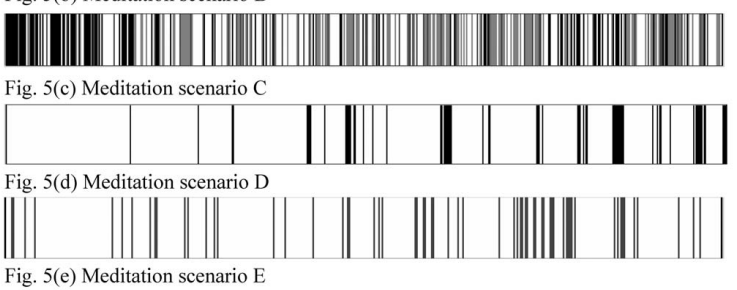

Fig. 5(f) Control group scenario

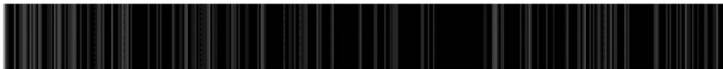

Fig.5 Five meditation scenarios based on evolution of meditation EEG illustrated by the running grayscale chart. The gray-scales from the darkest to the brightest colors indicate, respectively, the $\alpha^{+}, \Delta$, $\Delta+\theta, \theta+\underline{\alpha}$, and $\Phi$ patterns.

\section{CONCLUSION}

As the transition of consciousness states in meditation is still a myth, this paper reports a novel idea of understanding various meditation scenarios via EEG interpretation. Instead of the $\theta$ trains and $a^{+}$ rhythms observed in the TM deep meditation [7], our finding shows that substantial EEG power suppression ( $\Phi$ prototype), sometimes accompanied with $\beta$ rhythms, represents the most significant feature characterizing the sacred, unified, egoless, and blessed meditation stage in the orthodox Zen-Buddhism group. Experimental subjects' narration further corroborates, from the macroscopic viewpoint, the results of EEG interpretation obtained by the FCM-merging strategies. Without pre-specified prototypical EEG features, the FCM clustering method automatically identifies the significant features to be used as the meditation EEG interpreting protocol. Sophisticated merging strategies, developed according to the experiences of naked-eye EEG interpretation, further compensate for the intrinsic deficiency of background knowledge and bring the interpretations by the human and the computer closer together.

\section{REFERENCES}

1. Anand BK, Chhina GS and Singh B: Some aspects of electroencephalographic studies in yogis. Electroenceph clin Neurophysiol 1961; 13: 452456.

2. Wallace RK: Physiological effects of transcendental meditation. Science 1970; 167: 1751-1754.

3. Banquet JP: Spectral analysis of the EEG in meditation. Electroenceph clin Neurophysiol 1973; 35: 143-151.

4. Williams P and West M: EEG responses to photic stimulation in persons experienced at meditation. Electroenceph clin Neurophysiol 1975; 39: 519522.

5. West MA: Meditation and the EEG. Psychol Med 1980; 10: 369-375.

6 . Becker DE and Shapiro D: Physiological responses to clicks during zen, yoga, and TM meditation. Psychophysiology 1981; 18(6): 694-699.

7 . Jevning R, Wallace RK and Beidebach M: The physiology of meditation: A review. A wakeful hypometabolic integrated response. Neurosci biobehav rev 1992; 16: 415-424.

8. Aftanas LI and Golocheikine SA: Human anterior and frontal midline theta and lower alpha reflect emotionally positive state and internalized attention: high-resolution EEG investigation of meditation. Neurosci lett 2001; 310(1): 57-60.

9. Lo PC and Leu JS: Adaptive baseline correction of meditation EEG. Am J Electroneurod T 2001; 41(2): 142-155. 23

10. Travis F, Arenander A and DuBois D: Psychological and physiological characteristics of a proposed object-referral/self-referral continuum of self-awareness. Conscious Cogn 2004 Jun; 13 (2): 401-420.

11. Kim YY, Choi JM, Kim SJ, Park SK, Lee SH and Lee HK: Changes in EEG of children during brain respiration-training. Am J Chin Med 2002; 30 (23): 405-417.

12. Lo PC, Huang ML and Chang KM: EEG Alpha Blocking Correlated with Perception of Inner Light During Zen Meditation. Am J Chin Med 2003; 31(4): 629-642.

13. Cooper R, Osselton JW and Shaw JC: EEG Technology. 3rd ed, Butterworth Inc., Woburn, MA, 1980.

14. Smith JR: Automated analysis of sleep EEG data, Handbook of Electroencephalography and Clinical Neurophysiology, 2 (chapter 4), Lopes Da Silva FH et al. ed., Elsevier Science Publishers, 1986.

15. Niedermeyer E and Lopes Da Silva FH. Electroencephalography: Basic Principles,Clinical 
Applications,and Related Fields. 4th ed., Williams and Wilkins, USA, 1999.

16. Aldroubi A and Unser M: Wavelets in medicine and biology. CRC, Boca, Raton 1996.

17. Akay M: Wavelet applications in medicine. IEEE Spectrum 1997 May; 34(5): 50-56.

18. Daubechies I: Where do wavelets come from? A personal point of view. Proceedings of the IEEE 1996 April; 84(4): 510-513.24

19. Mallat SG: A theory for multiresolution signal decomposition: the wavelet representation. IEEE Trans on Pattern Anal and Machine Intell 1989 July; 11(7): 674-693.

20 . Cardenas-Barrera JL, Lorenzo-Ginori JV and Rodriguez-Valdivia EA: Wavelet-packets based algorithm for EEG signal compression. Med Inform Internet 2004 Mar; 29 (1): 15-27.

21. Ciaccio EJ, Dunn SM and Akay M: Biosignal pattern recognition and interpretation systems. 3 . Methods of classification. IEEE Eng Med Biol Mag 1994; 13(1): 129-135.

22 . Geva AB and Kerem DH: Forecasting generalized epileptic seizures from the EEG signal by wavelet analysis and dynamic unsupervised fuzzy clustering. IEEE Trans Biomed Eng 1998 Oct; 45(10): 1205-1216.

23. Bezdek JC: Pattern Recognition with Fuzzy Objective Function Algorithms. New York: Plenum, 1981.25 